\title{
Nationality and Intersubjectivity of Transnational Chinese Cinema
}

\author{
Chen Xiaokang ${ }^{1}$ \\ ${ }^{1}$ Department of Chinese, Shengli College China University of Petrol, Dongying, Shandong, China \\ Email: happy_xiaokang@163.com
}

\begin{abstract}
The nationality in transnational Chinese cinema are always been expressed in two ways: one is to highlight it and become the mirror image of the Western expression; the other is to conceal it and become the aphasic subject under the Hollywood paradigm. The explicit or implicit national expression is to seek a business opportunity, but it couldn't avoid the cost of cultural discount. The strategy to get out of this dilemma is to construct a kind of "transnational subjectivity", which is similar to intersubjectivity. On the premise of recognizing and respecting differences, it will reshape national character without losing borders in equal dialogue relations. The way to construct the transnational subjectivity is to seek the "free expression" of national character, and it depends on the prosperous local film market and highly conscious of cultural identity.
\end{abstract}

Keywords: Transnational Chinese cinema, nationality, transnational subjectivity

\section{跨国华语电影的民族性与主体间性}

\author{
陈小康 ${ }^{1,}$ \\ 中国石油大学胜利学院中文系, 东营, 山东, 中国 \\ happy_xiaokang@163.com

\section{摘要} \\ 跨国华语电影的民族性表达呈现出两种趋势：一是彰显之，成为西方表述中的 “他者” 镜像; 二是隐 \\ 匿之，成为 “好莱坞范式”之下失语的主体。或显或隐的民族性的表达是为了寻求一种商机，然而却 \\ 要以文化的折扣为其代价。走出这一困境的策略是建构一种类似于主体间性的 “跨国主体性”，以承认 \\ 并尊重差异为前提，在平等的对话关系中使民族性得以重塑却又不失边界。建构跨国主体性的途径在 \\ 于谋求民族性的 “自在的表达”，而其最根本的依托则是繁荣的本土电影市场和高度自觉的文化认同。 \\ 关键词：跨国华语电影；民族性；跨国主体性；
}

\section{1. 前言}

近年来, 以合拍片为代表的跨国电影以其充足的资 金和广阔的市场成为备受欢迎的影片制作形式。迄今为 止我国已和包括法国、英国、韩国、西班牙在内的 14 个 国家（地区）签订了电影联合摄制协议，国家电影局批 准立项的合拍片逐年上升。由此可见, 华语电影在资本、 制作、发行、放映等领域展开了前所未有的全球流动和 跨国想象。一个随之而来的问题是: 这些跨文化、跨国 家 (地域) 的电影在多大程度上是中国电影? 换言之, 其民族性是如何进行自我表达的, 中国作为主体的身份 在跨文化语境中又是如何呈现的呢?

\section{2. 跨国语境中民族性何以可能}

在民族电影的研究范式中，民族性是一个不言自明 的在场, 它是一个民族的语言、风俗、审美、价值观念 与宗教信仰的总和，是民族电影的身份得以被感知、被 识别、被认同的依据。但在全球化时代，民族性的合法 性遭到质疑: 所谓的全球化难道不是以民族性和现代民 族国家的消亡为前提吗? 然而事实并非如此。在《跨国 华语电影的民族性: 反抗与主体性》一文中，英国学者 裴开瑞以美国 “咄咄逼人的外交政策” 为据否定了现代 民族国家的颓势。他声称: “民族性不但在我们这个跨国 时代里依然生生不息, 在跨国电影研究中, 它同样是一 个有着长久学术生命的研究对象。” [1] 不仅如此, 全球 化和民族性还是一对矛盾而又彼此依存概念：正是由于 全球化的到来，民族性的问题才得以凸显。著名汉学家 
宇文所安曾以诗人杜甫为例来说明全球化与民族性的 关系: “在他变成一个伟大的中国诗人之前, 杜甫仅是一 个伟大的诗人而已。” ${ }^{[2]}$ 可见，民族性并非自古有之， 至少在全球化时代到来之前, 它尚不能被清晰地指认。 如果民族是一个想象的共同体, 那么这一对自我或他者 的想象显然是随着跨国现象的产生而开启, 并随着全球 化时代的到来而日鉌完善。恰恰是在跨国语境中, 在与 其它民族国家之间形成的镜像关系中, 民族性建构了自 我。回到电影上来: 跨国语境中的民族性是如何呈现自 己的呢? 总体来说可分为两种趋势: “他者化” 与 “好莱 坞化”。

\section{3. 想象的中国: 西方表述中的 “他者” 镜像}

作为一种市场策略, 早期征战好莱坞的跨国华语电 影多用各种代表中华文化的标志来凸显其民族性。例如 民俗风情、中国功夫和武侠世界。然而正如萨义德在他 的著作《东方学》中所指出的: “东方几乎是一个欧洲人 的发明, 它自古以来就是一个充满浪漫传奇色彩和异国 情调的、蒙绕着人们记忆和视野的、有着奇特经历的地

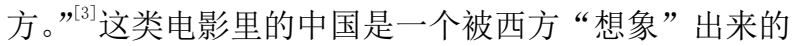
东方古老国度, 作为 “他者” 来实现对西方自身的识别 与认同, 其民族性的表达带有鲜明的西方中心主义色彩。 下文将通过几种不同类型的影片加以说明。

功夫片这一从李小龙时代被建构起来的文化符号, 至今仍是跨国语境中最典型的中国形象的表征, 但影片 呈现的中国想象往往带有鲜明的西方视角。在成龙的影 片《功夫梦》 (2010 年) 里, 美国少年帕克第一天到中 国，就因莫须有的原因被一位凶狠的中国少年拳脚相加。 而这位中国少年颈上的红领巾成为片中最为强烈的意 识形态符号, 此时的他们俨然上世纪 70 年代狂热的红 卫兵。由此, 一个冷漠、残忍、暴戻、专制的社会主义 中国, 再一次作为 “他者” 被西方世界所指认, 用以完 满其对自身进步、民主和文明的想象。影片在某种程度 上展现了当下中国在全球化时代的文化处境: 西方社会 对于中国的想象未曾随着时代的变迁而改变, 它像是一 个被定格的镜头, 一个被固定的标本, 恒久地停留在了 上世纪七十年代。武侠片是另一类在国际市场大受欢迎 的类型片。它同样是以功夫作为其民族性表征的, 但又 不限于此。以李安的《卧虎藏龙》(2000 年)、张艺谋的 《英雄》(2002 年) 为代表的影片致力于建构一个诗意 的武侠世界。它以江湖恩仇作为叙事的基本线索, 塑造 浪漫、感伤的江湖儿女, 传达东方式的哲理幽思。大漠 斜阳、冷月劲竹、勾栏市井、晨钟暮鼓等传达中国式意 境的文化符号蕴含其中。与功夫片中的颇具真实性的武 打动作相比, 武侠片的动作设计往往更加轻盈飘逸、潇 洒舒展, 更具东方写意美学的特征。在影片《卧虎藏龙》 中, 李安创造了是一个想象的、乌托邦式的中国, 它的 意象与美学意味彰显着中国文人独有的精神家园。与前 文所阐述的功夫片相比, 它作为奇观的成分似乎没那么 显著, 更多地是在进行自我言说而非被言说。“他性” 的成分有所弱化, “我性” 成分增加了。但是由于跨国
资本自身的逻辑，这类影片在发行的时候，依旧以异国 情调作为主要卖点, 再辅以西方本土文化的符号与普适 性价值观。《卧虎藏龙》的宣传语包括: “简・奥斯汀与 李小龙结合后产生的新品”、“功夫泰坦尼克”。尽管 影片的制作者并没有刻意地贩卖异国情调，但 “古代中 国”、“女性主义” 叙事的设定，使得影片在接受层面不 可能完全摆脱来自西方的审视的目光。

如果说上述影片所呈现的民族性尚有进行自我表 述的欲望, 那么部分以民俗风情为表现内容的电影则彻 底放弃了这一欲望, 它们甘于 “被表述”, 从而落入了 “自我东方化”或 “自我他者化” 的陷阱。《雪花秘扇》 就是一部典型的关于 “东方奇观” 的影片。影片对 “老 同” 这一民俗进行了渲染，但暧昧的情欲镜头使姐妹情 谊不可避免地流向女同性恋的隐蔽叙事。片中出现的缠 足、女书、鸦片、瘟疫、战乱、婚嫁习俗等文化符号, 几乎涵盖了所有固化的、西方式的中国想象，但所有的 元素又都点到为止, 丧失了挖掘更深的文化意蕴的可能。 裹脚布上渗出的鲜血, 骨头被折断时发出的声响, 显示 出“裹脚” 对人性的摧残, 但 “裹脚” 这件事情却又让 两位女主角的一生“被裹在一起”, 从而使这一泯灭人性 的习俗被赋予了浪漫感伤的维度, “奇观” 性不言而喻。 这部由中美双方联合制片的电影是一部标准的合拍片, 其导演王颖是美籍华人, 编剧阵容大多是美国人, 主要 演员李冰冰和全智贤分别来自中韩两国，因而注定是一 个异质文化交汇的复杂文本, 多种话语声音形成轰鸣的 混响。在这一系列话语中, 中国文化的声音十分微弱, 它仅仅在做浅表性的表演，得不到自我表述的机会，从 而丧失了与西方文化对话的可能。

如此便陷入了一个僵局: 似乎跨国电影中任何对于 民族性的言说都必然是西方视角下的奇观。对于像中 国这样的非西方国家来说，打破这一僵局何其困难：对 非西方世界的异国情调化不正是西方文化帝国主义事 业的一部分吗? 华语电影为了寻求商机, 不得不进入这 一后殖民主义的图景之中，以文化折扣换取商业利润。 讽刺的是, 这类以贩卖异国情调作为策略的电影并没有 得到商业上的巨大成功, 持久而固化的东方形象也给西 方观众带来了审美疲劳, 除了《卧虎藏龙》《英雄》等佼 佼者之外, 大多票房惨淡, 就连获得过巨大成就的成龙, 也一度选择回归香港和内地市场。这不得不说是一种文 化和商业的双重失败, 显示了跨国语境里中国主体性的 式微。

\section{4. 消失的中国：好莱坞范式下的主体失语}

如果说作为 “他者” 的华语电影是以 “民族性的彰 显” 融入跨国市场, 从而显示了中国主体性的式微, 那 么此类以 “民族性的隐匿” 为策略的华语电影, 则构成 了对中国主体性身份的更大的挑战。这类影片在运作形 式、内容表述和价值观念方面呈现出鲜明的“好莱坞化” 特征。好莱坞作为全球最大的电影工业体系, 凭借其高 度类型化的影片模式和成熟的商业运作体系席卷全球, 开拓了巨大的海外市场。面对激烈的市场竞争，华语电 
影的从业者们试图通过对好莱坞的学习和借鉴来增强 自身的海外传播能力, 于是出现了一批以好莱坞式的制 作方式、美学风格和价值观念为标尺的华语电影。其最 为显著的特征是对大片模式的效仿和对西方价值观念 的认同。

自张艺谋的《英雄》(2002 年) 开启了中国电影的 大片时代，高成本、大制作的华语大片不断涌现在我们 的视野中, 如《十面埋伏》 (2004 年) 《无极》 (2005 年)

《夜宴》 $(2006$ 年) 《满城尽带黄金甲》 $(2006$ 年) 《赤 壁》(2008 年)《金陵十三钗》(2011 年)《太平轮》(2015 年)《长城》(2017 年) 等, 此类影片多以规模宏大、制 作精良的视觉效果而著称, 往往有大牌影星的加盟, 并 面向全球发行。尽管这类影片一度被认为是中国电影 “走出去” 的希望所在, 是全球化时代对抗好莱坞电影 的中国骄傲, 但一个不容忽视的问题是, 以这样一种拍 摄形式获得成功恰恰表现出对好莱坞所制定的跨国电 影工业准则的臣服。且许多大片在追求类型化的同时也 放弃了对中国本土的人文关怀，除《金陵十三钗》和《太 平轮》呈现出了对中国近代历史的关心，又有哪一部涉 及到了中国人真实的困境和焦虑呢? 虽然繁复的中国 文化元素充斥其中, 但 “中国” 却成为一个渐渐模糊的 能指, 失去了它的与在地性与当下性。

除了对大片模式的效仿, 跨国华语电影也偏爱这样 一种暧昧性的表述方式: 用西方式的镜像表达一个与中 国有关的故事, 其本质则是用中国元素的外衣包裹西方 价值观念的内核。这一策略是以增强华语电影的海外传 播能力为目的, 但异质文化的融合并非易事, 稍有偏差 就使得影片面临一种文化的若境与价值观念的偏离。例 如, 影片《赤壁》把一场有着丰厚的历史底蕴和丰满的 人物形象的赤壁之战表述为一场发生在中华大地上的 特洛伊之战一一曹操进攻江东的野心竟是 “为了一个女 人”。导演吴宇森曾多次提及, 他要拍一个 “国际化” 的 三国, 他说: “我觉得, 三国, 故事应该是像奥运精神一 样, 团结、坚毅、勇气, 还有和平竞争、和平生活的气 息和人与人之间真正的友谊。” ${ }^{[4]}$ 这样一种所谓 “国际 化” ，无非是 “去中国化” 的代名词。三国时期的波诡 云谲的政治斗争、波澜壮阔的历史画面、就这样不加质 疑和批判地用一种现代化、西方化的情感和价值观念所 阐释, 这显然跳出了中国式的历史言说和人文传统的范 型。我们也在其它一些小成本制作中看到了这一 “去中 国化” 的倾向。极具好莱坞色彩爱情轻喜剧《北京遇上 西雅图》同样在价值观念上向西方倾斜。两部影片都是 关于中国人在异国他乡的故事, 但影片中的中国恰恰成 为主人公们并不幸福的 “前史” 的发生地, 似乎只有西 方世界的浪漫爱情之都才能体味人生的真谛、获得灵魂 的救赎。这里暗合了好莱坞影片中对于西方文化尤其是 美国文化的高度认同，中国主体性的式微是不言而喻的。 学者贾磊磊曾说: “我们在本土电影市场上经过激烈的 市场争夺而站住脚的中国电影, 在价值取向上又回到了 美国的文化版图内, 这不能不说是中国电影心理市场的 一种严重流失。”

盲目的好莱坞崇拜无疑使得中国电影主体性遭到 了空前的挑战。“他者化”的华语电影尚且有一个想象的
中国可以被指认（尽管这一想象带有某种程度上的文化 虚假), 而 “好莱坞化” 的华语电影却只留给我们一个身 份暧昧、面容模糊的中国，它在某种程度上是 “去中国 化” 的代名词。诚然，“好莱坞化”、“去中国化” 都是跨 国华语电影增强海外传播能力的策略, 似乎唯有如此, 才能构筑一种全球性的华语电影，从而被来自不同地域、 不同种族、不同文化背景的观众所接受。但这其中隐含 了两个预设的前提：一是中国性与全球性的二元对立， 二是 “好莱坞化” 与全球化的同一性。这一带有鲜明的 文化帝国主义色彩的话语在悄无声息地将中国和华语 电影推向边缘，并用自己的实践证实了文化霸权在这一 跨国时代的生生不息。好莱坞电影之所以能在跨国市场 一手遮天, 其根本原因是资本力量的强大。张艺谋在接 受媒体采访时曾说: “好莱坞从世界各地挖掘人才, 以高 额的薪水吸引他们来到美国。……在很多国家, 一旦导 演在本国获得名声，他很快就会被好莱坞挖掘。在汇集 资源和获取利润方面没有人能和好莱坞抗衡……" 资本吸引人才, 以人才保障高质量的电影作品, 好莱坞 由此称霸全球。但全球性电影绝非仅此一种可能。在好 莱坞之外, 欧洲电影的艺术坚守、韩国电影的本土追求、 伊朗电影独特视角都令我们深深折服, 虽然资本汇聚力 量不及好莱坞, 但并不妨碍其价值观念、审美趣味得到 有效的跨文化传播。相比之下，中国电影缺乏的是对本 土生活的热忱、对当下生活的关照, 而这样一种主体失 语将使得华语电影在未来的文化格局中失去自己的生 存空间。当然, 这里并非全盘否认华语电影对好莱坞范 式的推崇, 而是表达一种对多元化的期待: 当我们想看 一个有着深厚中国传统的电影时, 至少还能有的选。

\section{5. 一种策略：跨国主体性的建构与民族性的 “自在的表达”}

在华语电影或隐或显的民族性的表达中，我们看到 其参与跨国秩序建构的艰辛, 这似乎是中国这样的弱势 文化国家与西方对话的必然过程。但我们无法规避这一 过程, 拒绝对话只能使自己落入固步自封的 “文化割据 主义”。面对跨国华语电影文化价值的失落, 我们迫切 需要一种文化自觉，从而在复杂的全球化语境中为民族 性的表达赢得空间。然而, 中华文化的本质是什么? 我 们至今也很难给出一个确切的答案。任何源远流长的文 化传统都是复杂多变的。尤其像中国这样的后发性国家, 近代以来的种种革命和运动, 从洋务运动、戊戌变法、 辛亥革命到五四新文化运动, 从抗日战争到新中国的成 立, 从文化大革命到改革开放, 在种种强势的外来话语 影响之下，中华文化几经修正、扬弃乃至推倒重来，在 复杂的历史语境中不断进行自我重构。每一次重构, 都 是其与异质文化交汇、融合的结果。如果用发展的眼光 看待中华文化, 就会发现民族性并非某种 “至为根本而 又一成不变的质素” ${ }^{[7]}$ ，它不断敞开并将永远处于建构 之中。然而民族性又并非全然失去边界, 它必须有所坚 守从而避免成为一个空泛的能指。因此, 跨国华语电影 的主体性实则是一种 “跨国主体性” , 它类似于胡塞尔 
所提出的 “主体间性” (intersubjectivity), 即承认 一个主体间的共同世界的存在, 承认 “他者” 是与 “自 我” 并存的主体, 它们平等、共生, 在交互作用中获得 对彼此的确认。跨国主体性建构以承认差异、尊重他者 为前提寻求一种深层的对话。在这场异质文化的对话中, 民族性将不断地重构自我, 从而在具有群体性、社会性、 普遍性、世界性的文化场域中召唤一个主体。由于一个 先验的共同体世界的存在，对 “自我” 和 “他者” 的通 达并非以一方对另一方的征服为途径, 而是二者在交互 作用中彼此照亮。“差异”正是跨国主体性得以生成的根 本，这就为民族性的自我重构设置了边界。至于这一边 界究竟是什么, 在当下语境中尚未得到清晰的言说。但 毫无疑问，它的存在确立了跨国主体性的合法。

跨国主体性的建构既不同于将自身“他者化”的“唯 差异式” 方法, 也不同于将自身 “好莱坞化” 的 “无差 异式” 策略, 而是在正视差异的前提下, 以对话的方式 寻求异质文化的可通约性。它通过民族性的 “自在的表 达” 建构自我, 既不迎合也不抵抗。那么, 怎样才称得 上一种 “自在的表达” 呢? 电影《长城》和动画电影《白 蛇: 缘起》或许可以给我们一些启示。前者努力传达 “信 任”、“为国家人民而战” 等中国式的精神美德, 但在嵌 入文本的过程中显示出深深的无力感, 并表现出与故事 本身的疏离一一主人公的价值认同转变是如此的生硬, 以至于观众难以认同。叙事逻辑的硬伤只是表层现象, 其根本原因在于面对西方强势文化时主体性的焦虑。为 了彰显主体性, 便将价值观念强行赋予一个并不足以承 载它的文本之中。当文本本身不能发出这样的声音时, 作者则开始代替它发出声音, 影片由此呈现崩坏之势。 这一紧张的言说方式, 背离了民族性“自在的表达”, 从 而失去了建构有效的跨国主体性的可能。《白蛇: 缘起》 虽只是一部小成本动画电影, 但却呈现了本土化的内容 和国际化的叙事。它让观众沉醉在充满古风的美术造型 与似曾相识的民间传说之中, 但其主题意蕴和叙事节奏 却是好莱坞式的。它没有沉重的话语负担, 故事讲得轻 盈而自在。因此, 在所有民族性的表达中, 唯有 “自在 的表达” 才可能建构有效的跨国主体性, 其前提则是摆 脱主体性的焦虑, 避免将自我和他者设定为紧张对抗的 二元对立关系。由此也可探知 “他者化”、“好莱坞化” 的华语电影文化上失败的原因, 不论是民族性的彰显或 隐逸, 其根本立场均是将自身与西方文化对立, 寻求民 族性的 “刻意的输出” 或 “刻意的遮蔽”, 放弃了主体间 性的思维方式, 并因中国文化的弱势而表现出了对好莱 坞工业秩序的臣服。

这种民族性的 “自在的表达” 究竟是不是一种乌托 邦? 我们只需注意到, 在跨国语境中, 欧洲电影依旧保 持着坚定艺术立场, 韩国电影依旧保持着深刻的人文思 考, 伊朗电影依旧探索着温情的现实人生, 印度电影依 旧呈现着欢乐的民族歌舞, 就应当确信这一 “自在的表 达” 的存在。而民族性 “自在的表达” 必须依托于的高 度的文化自信与深刻的文化自省，否则只能是空中楼阁。 在全球化时代到来之前, 我们也曾有《霸王别姬》这样 的底蕴深厚的电影, 也曾有《阳光灿烂的日子》这样激 情洋溢的电影, 彼时的中国远没有达到今天的经济地位,
但文化自省与自信的程度却是今天所不可及的。时至今 日, 我们已初步具备了与西方对话的可能, 但跨国主体 性的建构依旧要立足本土, 一个繁荣的电影市场和自觉 的文化认同是这一诉求的全部立场所在。全球化是挑战, 更是机遇，我们有理由期待中国电影美好的未来。

\section{项目基金}

本文为山东省艺术科学重点课题《新世纪 以来华语电影的民族主义叙事与国家形象建 构》（201806144）的阶段性成果之一。

\section{REFERENCES}

[1] Berry, C. (2006) Nationality in transnational Chinese films: Resistance and subjectivity. World Cinema, 1: 4

[2] Owen, S.O. (2003) Borrowed Stone: Stephen Owen's Selected Essays. JSPPS, Nanjing.

[3] Said, E.W. (1999) Orientalism. SDX Joint Publishing Company, Beijing.

[4] Meng, J. (2006). Wu Yusen: This is a worldwide Sanguo.

http://www.lifeweek.com.cn/2008/0701/22062.shtml

[5] Jia, L.L. (2013). Slanting sky: The cultural orientation of Finding Mr. Right. Art Criticism, 6: 62

[6] Zhu, Y. (2008). Global Chinese films and Hollywood blockbusters. World Cinema, 3: 57

[7] Berry, C. (2006) Nationality in transnational Chinese films: Resistance and subjectivity. World Cinema, 1:5 